\title{
Near-drowning-associated pneumonia with bacteremia caused by coinfection with methicillin-susceptible Staphylococcus aureus and Edwardsiella tarda in a healthy white man: a case report
}

\author{
Lucas Santos Zambon*, Guilherme Nader Marta, Natan Chehter, Luis Guilherme Del Nero \\ and Marina Costa Cavallaro
}

\begin{abstract}
Background: Edwardsiella tarda is an Enterobacteriaceae found in aquatic environments. Extraintestinal infections caused by Edwardsiella tarda in humans are rare and occur in the presence of some risk factors. As far as we know, this is the first case of near-drowning-associated pneumonia with bacteremia caused by coinfection with methicillin-susceptible Staphylococcus aureus and Edwardsiella tarda in a healthy patient.
\end{abstract}

Case presentation: A 27-year-old previously healthy white man had an episode of fresh water drowning after acute alcohol consumption. Edwardsiella tarda and methicillin-sensitive Staphylococcus aureus were isolated in both tracheal aspirate cultures and blood cultures.

Conclusion: This case shows that Edwardsiella tarda is an important pathogen in near drowning even in healthy individuals, and not only in the presence of risk factors, as previously known.

Keywords: Near drowning, Edwardsiella tarda, Pneumonia, Bacterial, Bacteremia

\section{Background}

The World Health Organization defines drowning as "the process of experiencing respiratory impairment from submersion/immersion in liquid" [1] emphasizing the importance of respiratory system damage in drowning pathophysiology, complications, and prognosis. More than 500,000 people die each year due to unintentional drowning [2]. According to the Center for Diseases Control, drowning was the tenth major cause of death related to injuries in the USA from 1999 to 2010 [3]. Approximately $50 \%$ of drowning victims are under 20 -years old [4]. In developing countries this incidence is even greater [5].

\footnotetext{
* Correspondence: lucaszambon@yahoo.com.br

Emergency Department, Hospital das Clínicas da Faculdade de Medicina da Universidade de São Paulo, Av. Dr. Enéas de Carvalho Aguiar, 255, 50 andar, Sala 5023, Cerqueira Cesar, ZIP Code 05403-010 São Paulo, SP, Brazil
}

Lung infections are one of the most serious complications occurring in victims of drowning [6]. They may represent a diagnostic challenge as the presence of water in the lungs hinders the interpretation of radiographic images [5]. Both fungi and bacteria have been reported as etiological agents of after-drowning pulmonary infections [6]. Aerobic Gram-negative bacteria are the most frequently implicated in these infections [6].

Edwardsiella tarda is a facultative anaerobic flagellated Gram-negative bacilli member of the Enterobacteriaceae family found in aquatic environments [7]. This bacteria causes gastroenteritis predominantly. The main risk factors for extraintestinal infections are hepatobiliary diseases, iron overload syndromes, cancer, immunosuppression, and diabetes mellitus [8, 9].

As far as we know, the case about to be presented is the first documented episode of near-drowning-associated 
pneumonia with bacteremia caused by coinfection with methicillin-susceptible Staphylococcus aureus and E. tarda in a healthy patient. These data could motivate a different approach to antibiotic use for sepsis related to a neardrowning episode.

\section{Case presentation}

A 27-year-old previously healthy white man had an episode of fresh water drowning after acute alcohol consumption. Friends quickly removed him from the water. A rescue team was activated and identified cardiopulmonary arrest in a non-shockable rhythm. Oral intubation was quickly performed. Neither stool reflux/vomiting nor aspiration was reported by the team. After two cycles of cardiopulmonary resuscitation (for about 4 minutes) and orotracheal intubation, return of spontaneous circulation occurred. During transportation bradycardia was reported, which reverted after one dose of atropine.

He was admitted to the emergency room of a tertiary academic hospital. On examination he was hemodynamically stable and comatose with 3 points on Glasgow Coma Scale (GCS) and nonreactive pupils. No other relevant physical findings upon arrival. He was placed on mechanical ventilation and transferred to the intensive care unit (ICU).

A few hours after admission to the ICU he presented decreased consciousness level (GCS 4), hypotension, and signs of poor peripheral perfusion. A blood gas analysis showed hypoxemia with respiratory acidosis. He underwent hypothermia for neuroprotection after cardiac arrest, received protective ventilation for acute respiratory distress syndrome (ARDS), and vasoactive drugs (norepinephrine plus epinephrine, which were maintained for 24 hours) through right subclavian central venous catheter (postpuncture pneumothorax was drained with a pigtail catheter uneventfully). He developed acute renal failure due to rhabdomyolysis, renal ischemia, and multiple organ failure, requiring hemodialysis for 15 days.

Gram's staining of his tracheal aspirate taken 3 days after the accident showed Gram-positive cocci isolated and in pairs, and frequent Gram-negative bacilli. Tracheal aspirate cultures isolated methicillin-sensitive $S$. aureus, Enterobacter aerogenes, Aeromonas species, and E. tarda. Blood cultures (first set obtained) isolated methicillin-sensitive S. aureus and E. tarda, which led to the introduction of oxacillin and ceftriaxone on the sixth day of hospitalization. Five more sets of blood culture were performed after the introduction of the antibiotics. All were negative. Computed tomography performed on the 11th day of hospitalization showed bilateral pleural effusion, and multiple pulmonary consolidations and cavities with thickened walls and air-fluid levels, consistent with lung abscesses (Figs. 1 and 2).

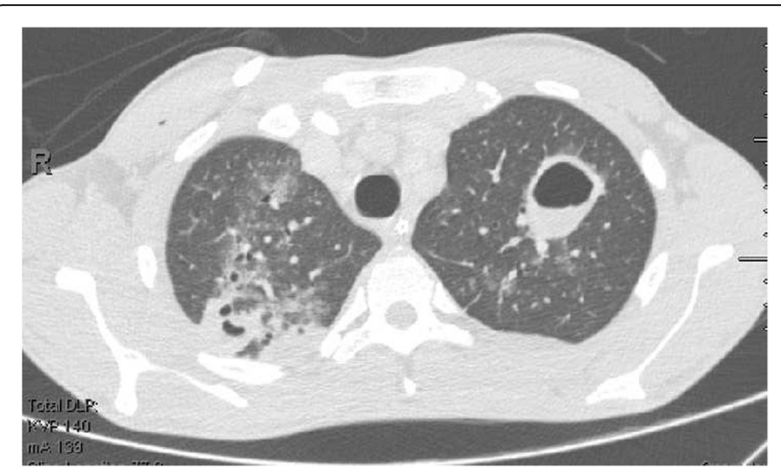

Fig. 1 Upper third of the chest in computed tomography scan performed on the 11th day of hospitalization showed foci of pulmonary consolidation and cavities with thickened walls and fluid levels

Twenty days after the ICU admission, he was transferred to the regular infirmary ward where the ongoing clinical, laboratory, and radiological improvement continued. On the 45th day of hospitalization, he was discharged home for out-patient monitoring with prescription of ciprofloxacin and clindamycin to be taken orally. $\mathrm{He}$ returned for follow-up consultation 14 days after taking the antibiotics. He reported no symptoms since the hospital discharge.

\section{Discussion}

Although most victims of near drowning are previously healthy, the morbidity and mortality associated with these events are high, mainly due to pulmonary and

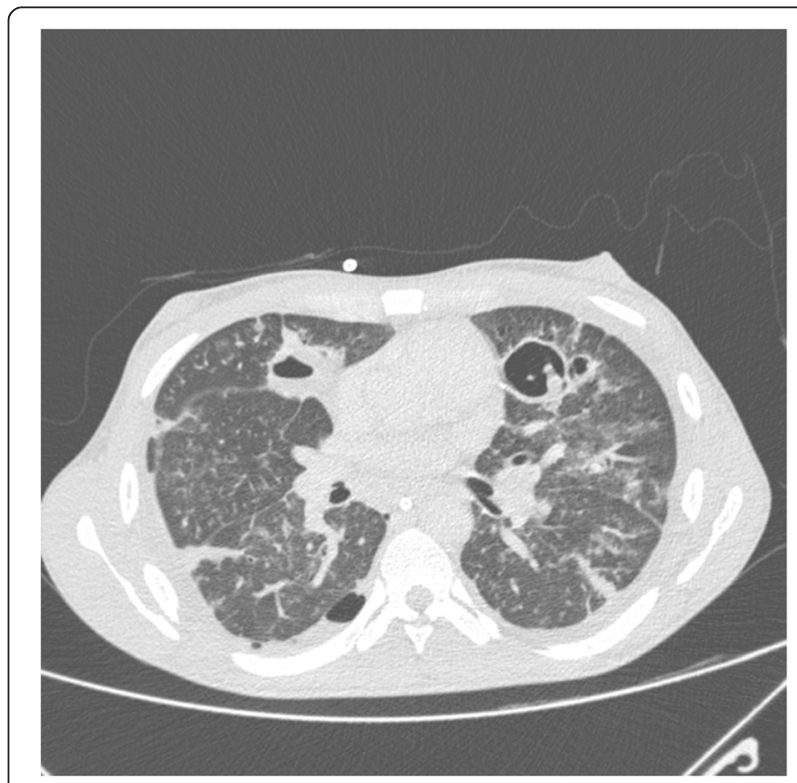

Fig. 2 Middle third of the chest in computed tomography scan performed on the 11th day of hospitalization showed foci of pulmonary consolidation and cavities with thickened walls and fluid levels 
neurological complications associated with tissue damage by hypoxia, acidosis, and hypoperfusion [6]. After submersion, the victim's conscious response leads to a period of voluntary apnea, which stimulates the respiratory drive, leading to involuntary aspiration [5]. Aspirated water, in contact with the alveoli, leads to surfactant dysfunction and an increase in the alveolarcapillary membrane permeability, causing extensive pulmonary edema, atelectasis, and bronchospasm [10]. The combined effects of alveolar damage, contaminated material inoculation in the airways, and the frequent need of mechanical ventilation respiratory support result in an up to $12 \%$ risk of after-drowning pneumonia [11]. This risk may vary according to the volume aspirated, the degree of water contamination and its temperature, as well as to the occurrence of aspiration of gastric content [6]. When admitted to an ICU, drowning victims should be managed following ARDS guidelines [5].

Lung infections are one of the most serious complications occurring in victims of drowning [6]. They may represent a diagnostic challenge as the presence of water in the lungs hinders the interpretation of radiographic images [5]. However, prophylactic antimicrobial therapy is not recommended due to the potential selection of resistant bacteria [12]. Both fungi and bacteria have been reported as etiological agents of after-drowning pulmonary infections [6]. Aerobic Gram-negative bacteria are the most frequently implicated bacteria in these infections, among which stand out Aeromonas species (in particular, Aeromonas hydrophila), Burkholderia pseudomallei, and Chromobacterium violaceum [6]. Grampositive cocci such as $S$. aureus and Streptococcus pneumoniae and some Enterobacteriaceae are also reported as etiological agents of pneumonia, although it is often difficult to distinguish whether the infection was due to drowning or nosocomial related [6].

E. tarda is a facultative anaerobic flagellated Gramnegative bacilli member of the Enterobacteriaceae family found in aquatic environments [7]. Pathogenicity in humans, although rare, has been demonstrated predominantly in gastroenteritis, which represents more than 80 $\%$ of the infections by this agent [7-13]. Nonetheless, there are also reports of extraintestinal infections such as cellulitis and cutaneous abscesses, meningitis, endocarditis, osteomyelitis, liver abscess, tubo-ovarian and peritoneal abscess, as well as bacteremia and sepsis $[8,9$, 11-14]. There is no report of pneumonia cases in immunocompetent patients so far. In the present case, only blood and tracheal aspirate cultures were performed. An endotoxin test was not available at the hospital. Nonetheless, the endotoxin is of secondary pathogenic importance when compared to infections caused by Salmonella, Shigella and Yersinia [15].
The most important risk factor for E. tarda infection is exposure to aquatic environments [13], and the main risk factors for extraintestinal infections are hepatobiliary diseases, iron overload syndromes, cancer, immunosuppression, and diabetes mellitus $[8,9]$.

\section{Conclusions}

This is the first report of near-drowning-associated pneumonia with bacteremia by coinfection with methicillinsusceptible $S$. aureus and $E$. tarda in a patient without comorbidities, documented by isolation of the bacteria from blood cultures and in tracheal aspirate cultures. The only reported case of pneumonia caused by E. tarda (isolated only in sputum) occurred in a patient hospitalized for diabetic ketoacidosis, with no history of drowning [9]. There are no reports of pneumonia caused by E. tarda in a patient without previous medical history, nor reports of $E$. tarda bacteremia from pulmonary infection. The capacity of $E$. tarda to form abscesses in other parts of the body such as skin, ovaries, and liver has already been well documented $[8,9,14]$. This may suggest its involvement in the formation of extensive lung abscesses in this case in association with the $S$. aureus, although there are also no reports of such clinical presentation.

Although extraintestinal E. tarda infections are susceptible to most antibiotics that target Gram-negative bacteria, including $\beta$-lactams, aminoglycosides, quinolones, tetracyclines, and chloramphenicol $[9,13,16]$, the lethality of extraintestinal E. tarda infections is approximately $23 \%$ [8], reaching rates as high as $50 \%$ in cases of bacteremia [13].

This case widens the spectrum of extraintestinal presentations of $E$. tarda infection to include bacteremia from lung infection. Thus, the monitoring of drowning victims for pulmonary infection should be thorough and should always include sputum cultures to allow detection of waterborne bacteria which, although rarely isolated, can cause highly lethal infections.

\section{Abbreviations \\ ARDS, acute respiratory distress syndrome; GCS, Glasgow Coma Scale; ICU, intensive care unit}

\section{Acknowledgements}

The authors would like to thank the entire emergency department staff involved in the care of the patient, also the intensive care unit staff. We also would like to thank the Discipline of Emergency Medicine of the Hospital das Clínicas da Faculdade de Medicina da Universidade de São Paulo, especially Dr Heraldo Possolo (MD, PhD), and Professor Irineu Tadeu Velasco (MD, PhD).

\section{Authors' contributions}

GNM and LSZ analyzed and interpreted the patient data regarding the disease, and conducted patient care. GNM and LSZ collected patient data, and described it in the manuscript. NC, LGDN, and MCC performed literature review, and major contribution in writing the manuscript. All authors read and approved the final manuscript. 


\section{Competing interests}

The authors declare that they have no competing interests.

\section{Consent for publication}

Written informed consent was obtained from the patient for publication of this case report and accompanying images. A copy of the written consent is available for review by the Editor-in-Chief of this journal.

\section{Received: 13 August 2015 Accepted: 7 June 2016}

Published online: 16 July 2016

\section{References}

1. van Beeck EF, Branche CM, Szpilman D, Modell JH, Bierens JJ. A new definition of drowning: towards documentation and prevention of a global public health problem. Bull World Health Organ. 2005:83(11):853-6.

2. World Health Organization, Dept. of Injuries and Violence Prevention. The injury chart book: a graphical overview of the global burden of injuries. Geneva: World Health Organization; 2002. p. 76.

3. National Center for Injury Prevention and Control, Web-based Injury Statistics Query and Reporting System (WISQARS) [online] 2005 http://www. cdc.gov/HomeandRecreationalSafety/Water-Safety/. Accessed 26 Jul 2015.

4. Layon AJ, Modell JH. Drowning: Update 2009. Anesthesiology. 2009; 110(6):1390-401

5. Szpilman D, Bierens JJ, Handley AJ, Orlowski JP. Drowning. N Engl J Med. 2012;366(22):2102-10.

6. Ender PT, Dolan MJ. Pneumonia associated with near-drowning. Clin Infect Dis. 1997;25(4):896-907.

7. Spencer JD, Hastings MC, Rye AK, English BK, Ault BH. Gastroenteritis caused by Edwardsiella tarda in a pediatric renal transplant recipient. Pediatr Transplant. 2008;12(2):238-41.

8. Wang IK, Kuo HL, Chen YM, Lin CL, Chang HY, Chuang FR, et al. Extraintestinal manifestations of Edwardsiella tarda infection. Int J Clin Pract. 2005:59(8):917-21.

9. Nelson JJ, Nelson CA, Carter JE. Extraintestinal manifestations of Edwardsiella tarda infection: a 10-year retrospective review. J La State Med Soc. 2009; 161(2):103-6.

10. Orlowski JP, Abulleil MM, Phillips JM. The hemodynamic and cardiovascular effects of near-drowning in hypotonic, isotonic, or hypertonic solutions. Ann Emerg Med. 1989:18(10):1044-9.

11. van Berkel M, Bierens JJ, Lie RL, de Rooy TP, Kool L, van de Velde EA, et al. Pulmonary oedema, pneumonia and mortality in submersion victims; a retrospective study in 125 patients. Intensive Care Med. 1996;22(2):101-7.

12. Wood C. Towards evidence based emergency medicine: best BETs from the Manchester Royal Infirmary. BET 1: prophylactic antibiotics in near-drowning. Emerg Med J. 2010;27(5):393-4.

13. Janda JM, Abbott SL. Infections associated with the genus Edwardsiella: the role of Edwardsiella tarda in human disease. Clin Infect Dis. 1993;17(4):742-8.

14. Slaven EM, Lopez FA, Hart SM, Sanders CV. Myonecrosis caused by Edwardsiella tarda: a case report and case series of extraintestinal E. tarda infections. Clin Infect Dis. 2001:32(10):1430-3.

15. Bockemühl J, Aleksic V, Wokatsch R, Aleksic S. Pathogenicity tests with strains of Edwardsiella tarda: detection of a heat-stable enterotoxin. Zentralbl Bakteriol Mikrobiol Hyg A. 1983;255(4):464-71.

16. Wilson JP, Waterer RR, Wofford JD, Chapman SW. Serious infections with Edwardsiella tarda. A case report and review of the literature. Arch Intern Med. 1989;149(1):208-10

\section{Submit your next manuscript to BioMed Central and we will help you at every step:}

- We accept pre-submission inquiries

- Our selector tool helps you to find the most relevant journal

- We provide round the clock customer support

- Convenient online submission

- Thorough peer review

- Inclusion in PubMed and all major indexing services

- Maximum visibility for your research

Submit your manuscript at www.biomedcentral.com/submit 